\title{
"Bridge" Neoadjuvant Endocrine Therapy for Early Stage Breast Cancer Patients During COVID-19 at an Academic Hospital in NYC: Lessons Learned and Future Directions
}

\author{
Joshua Feinberg ${ }^{1}$, Cindy Cen $^{2}$, Freya Schnabel ${ }^{1}$, Sylvia Adams $^{3}$, Magdalena Plasilova ${ }^{1}$, Janet Yeh $^{1}$, \\ Marleen Meyers ${ }^{3}$, James Speyer ${ }^{3}$, Elliot Belenkov ${ }^{3}$, Maryann Kwa ${ }^{3}$, Yelena Novik ${ }^{3}$, Elena Katz ${ }^{3}$, \\ Amber Azniv Guth ${ }^{1, *}$
}

${ }^{1}$ Department of Surgery, Breast Surgery Section, NYU Langone Health, New York University, New York City, U.S.A

${ }^{2}$ Department of Surgery, Hofstra University, Northwell Health, New Hyde Park, New York, U.S.A

${ }^{3}$ Department of Medicine, Division of Medical Oncology, NYU Langone Health, New York University, New York City, U.S.A

Email address:

amber.guth@nyulangone.org (A. A. Guth)

${ }^{*}$ Corresponding author

\section{To cite this article:}

Joshua Feinberg, Cindy Cen, Freya Schnabel, Sylvia Adams, Magdalena Plasilova, Janet Yeh, Marleen Meyers, James Speyer, Elliot Belenkov, Maryann Kwa, Yelena Novik, Elena Katz, Amber Azniv Guth. "Bridge” Neoadjuvant Endocrine Therapy for Early Stage Breast Cancer Patients During COVID-19 at an Academic Hospital in NYC: Lessons Learned and Future Directions. International Journal of Clinical Oncology and Cancer Research. Vol. 6, No. 1, 2021, pp. 38-41. doi: 10.11648/j.ijcocr.20210601.16

Received: January 24, 2021; Accepted: February 23, 2021; Published: March 4, 2021

\begin{abstract}
Background: The COVID-19 pandemic presented a unique challenge to our healthcare system and rapidly changed the delivery of cancer care. During the height of the pandemic in New York State, non-emergent surgery, including cancer surgery, was postponed. For patients with hormone receptor positive breast cancer, "bridge" neoadjuvant hormonal therapy was initiated until surgery could be safely performed. Objective: We present our institutional experience with this approach and lessons learned, including oncologic outcomes and factors which may lead to increased medication compliance. Method: This is a single institution, retrospective, observational chart review. Using data from the NYU Perlmutter Cancer Center, we created a database of patients who were diagnosed with ER+ stage 0 , I, or II breast cancer and were offered preoperative endocrine therapy (tamoxifen or aromatase inhibitors) between March 12, 2020 and June 1, 2020. Variables collected included demographics, tumor characteristics, as well as the rates of medication uptake and compliance. Patients eligible for review were adult (age>18) males and females who had initial visits at our two cancer centers between March 12, 2020 and June 1, 2020, during the height of the COVID-19 pandemic in New York. Results: Of 192 newly diagnosed breast cancer patients seen at the NYU Perlmutter Cancer Center during the study time period, 136 (71\%) patients had early stage ER positive breast cancer. Forty-five patients (23\%) had not yet undergone surgery, and were recommended to receive presurgical hormonal therapy as a bridge. Average age: $60.5+/-13.8$ years old (range 31-89). Thirty-four patients (75.6\%) had invasive cancer, 8 had DCIS (17.8\%), 3 had DCIS with microinvasion (6.7\%). There were 9 patients $(20 \%)$ who did not take the medication. Thirty-six patients (80\%) took medication for an average of $43.6+/-27.3$ days (range 9-101 days) prior to surgery. Twenty-eight patients (77.8\%) took an aromatase inhibitor, and $8(22.2 \%)$ took tamoxifen. Forty-two patients have now undergone surgery $(93.3 \%)$ - the remaining patients include one who is refusing all treatment, one patient who relocated out of state, and another patient who has not yet scheduled surgery, but is still taking an aromatase inhibitor. Conclusion: Despite traditionally low uptake and adherence to endocrine therapy, acceptance of neo-adjuvant bridge therapy during the COVID-19 pandemic was high (80\%). We plan to investigate our patient population further, especially psychosocial and behavioral factors that influence willingness to take endocrine therapy - and apply these lessons to management of early-stage ER positive breast cancer.
\end{abstract}

Keywords: Early Stage Breast Cancer, COVID-19, Neoadjuvant Endocrine Therapy 


\section{Introduction}

The COVID-19 pandemic presented a unique challenge to our healthcare system and rapidly changed the delivery of cancer care. In March 2020, it became apparent that hospitals would rapidly convert to COVID units, requiring the conservation of hospital resources and postponement of all non-urgent surgery, including oncologic procedures. A cancer-induced immunocompromised state may make breast cancer patients particularly susceptible to viral infection, and cancer patients appear to have a two-fold increased risk of contracting SARS-CoV-2 over the general population, due to both malignancy itself as well as anti-cancer treatments [1]. The recommendations released by the COVID-19 Pandemic Breast Cancer Consortium have guided surgeons in treating this patient population while not compromising outcomes due to a delay in surgery [2]. For patients with estrogen receptor-positive $(\mathrm{ER}+)$ invasive and non-invasive cancers, the guidelines suggested bridging these patients with neoadjuvant endocrine therapy (NET) until surgery could safely be performed after the resolution of the pandemic.

The benefits of neoadjuvant systemic therapy include increased breast conservation rates and decreased rates of positive nodes. Additional advantages of pre-operative therapy include the ability to assess tumor response in vivo. In patients with ER positive disease, low pathologic complete response rates with neoadjuvant chemotherapy has argued against its use and has prompted studies assessing NET as an alternative treatment strategy in these patients [3]. The main benefit of NET seems to be the ability to convert the surgical option from mastectomy to breast conservation therapy. Rates of breast conserving surgery have been shown to be slightly higher with NET vs. neoadjuvant chemotherapy ( $33 \%$ vs $24 \%, \mathrm{P}=0.058$ ) [4]. NET is also better tolerated by patients than neoadjuvant chemotherapy [5]. A meta-analysis by Spring et. al. evaluated over 20 studies with 3,490 patients demonstrated NET is associated with equivalent response rates to neoadjuvant chemotherapy, but with lower toxicity [6].

Despite these findings, NET has been infrequently utilized in clinical practice [7]. NET has most commonly been used as an alternative to surgery in older patients $(>70)$ with hormone receptor positive breast cancer and significant comorbidities. Despite increased local failure rates, survival outcomes have been shown to be similar to neoadjuvant chemotherapy and, as such, NET is most often used in those patients with a limited life expectancy [4]. Currently, however, the COVID-19 pandemic has led to the utilization of NET in early stage, ER positive breast cancer and has presented a unique opportunity to study this underutilized treatment modality prospectively. To further explore this treatment strategy, we reviewed patients treated with NET at our institution in New York City, which was struck early and was at the epicenter of the crisis in the United States.

\section{Methods}

This is a single institution, retrospective, observational chart review. Using data from the NYU Perlmutter Cancer
Center, we created a database of patients who were diagnosed with ER+ stage 0 , I, or II breast cancer and were offered preoperative endocrine therapy (tamoxifen or aromatase inhibitors) between March 12, 2020 and June 1, 2020. Variables collected included demographics, tumor characteristics, as well as the rates of medication uptake and compliance. Patients eligible for review were adult (age $>18$ ) males and females who had initial visits at our two cancer centers between March 12, 2020 and June 1, 2020, during the height of the COVID-19 pandemic in New York. The database allows us to explore post-surgery outcomes as well as to gain insights into medication adherence. IRB approval was obtained for this study.

\section{Results}

Table 1. Patient Demographics and Tumor Characteristics.

\begin{tabular}{|c|c|c|}
\hline Variables & Total $(\mathrm{N}=45)$ & $\%$ \\
\hline Median Age (years) & $60.5(31-89)$ & \\
\hline \multicolumn{3}{|l|}{ Race } \\
\hline White & 26 & 57.8 \\
\hline Black & 12 & 26.7 \\
\hline Asian & 3 & 6.7 \\
\hline Other & 4 & 8.9 \\
\hline \multicolumn{3}{|l|}{ Menopause Status } \\
\hline Pre-menopausal & 10 & 22.2 \\
\hline Peri-menopausal & 1 & 2.2 \\
\hline Post-menopausal & 34 & 75.6 \\
\hline Mean BMI $\left(\mathrm{kg} / \mathrm{m}^{2}\right)$ & $28.3(17.8-46.5)$ & \\
\hline \multicolumn{3}{|l|}{ Palpability } \\
\hline Non-Palpable & 30 & 66.7 \\
\hline Palpable & 15 & 33.3 \\
\hline \multicolumn{3}{|l|}{ Mammographic Breast Density } \\
\hline Entirely Fatty & 2 & 4.4 \\
\hline Scattered Fibroglandular & 17 & 37.8 \\
\hline Heterogeneously Dense & 24 & 53.3 \\
\hline Extremely Dense & 2 & 4.4 \\
\hline \multicolumn{3}{|l|}{ Histology } \\
\hline DCIS & 8 & 17.8 \\
\hline DCIS with microinvasion & 3 & 6.7 \\
\hline Invasive carcinoma & 34 & 75.6 \\
\hline \multicolumn{3}{|l|}{ Clinical Stage } \\
\hline 0 & 8 & 17.8 \\
\hline I & 27 & 60 \\
\hline II & 10 & 22.2 \\
\hline Endocrine Therapy & $(\mathrm{N}=36)$ & \\
\hline Tamoxifen & 8 & 22.2 \\
\hline Aromatase Inhibitor & 28 & 77.8 \\
\hline Mean Duration of Endocrine Therapy (days) & $43.6(9-101)$ & \\
\hline
\end{tabular}

Of 192 newly diagnosed breast cancer patients seen at the NYU Perlmutter Cancer Center during the study time period, $136(71 \%)$ patients had early stage ER positive breast cancer (based on imaging, physical exam, and core biopsy results). Forty-five patients (23\%) had not yet undergone surgery, and were recommended to receive presurgical hormonal therapy as a bridge because of the COVID-19 pandemic (Table 1). The average age was $60.5+/-13.8$ years old (range $31-89$ ), and all were female. Thirty-four of 44 patients were post-menopausal (75.6\%), 10 patients were pre-menopausal $(22.2 \%)$ and one was peri-menopausal $(2.2 \%)$. Twenty-six patients were white (57.8\%), 12 were black (26.7\%) 3 were Asian (6.7\%), and 4 
were other $(8.9 \%)$. Thirty-four patients $(75.6 \%)$ had invasive cancer, 8 had DCIS $(17.8 \%), 3$ had DCIS with microinvasion $(6.7 \%)$. There were 9 patients $(20 \%)$ who did not take the medication for various reasons: 1 contracted COVID-19, 1 declined treatment, 1 decided to transfer care out of state, 1 preferred to take a homeopathic remedy instead of endocrine therapy, 1 preferred to wait for surgery without medication, and 4 were scheduled for surgery sooner than anticipated and never initiated medication. The remaining 36 patients $(80 \%)$ took medication for an average of $43.6+/-27.3$ days (range 9-101 days) prior to surgery. Twenty-eight patients $(77.8 \%)$ took an aromatase inhibitor, and $8(22.2 \%)$ took tamoxifen. Forty-two patients have now undergone surgery (93.3\%) - the remaining patients include one who is refusing all treatment, one patient who relocated out of state, and another patient who has not yet scheduled surgery, but is still taking an aromatase inhibitor.

\section{Discussion}

The requirement for the reallocation of resources and cancellation of non-emergency surgery during the COVID-19 pandemic forced changes in the initial management of breast cancer patients, away from the traditional "surgery first" approach [7]. Although NET had infrequently been used in younger ER positive DCIS and early stage invasive breast cancer, there is certainly a basis for this treatment strategy that has been demonstrated by recent studies.

Addressing the concern that delaying surgery has a negative impact on cancer outcomes, Minami et al. identified over 378,000 patients in the National Cancer Database (NCDB) with DCIS or ER positive cT1-2N0 breast cancer treated between 2010-2016, and evaluated the association between time to surgery and pathologic stage [8]. The authors found that increased time to surgery was associated with a small increase in pathological upstaging in DCIS patients, but did not have an impact on overall survival. In patients with cT1-2N0 disease, NET use did not have an impact on stage or overall survival, thus supporting the use of bridging ER positive patients to surgery during the pandemic.

The benefit of allowing less aggressive surgery (from mastectomy to breast conservation surgery) is demonstrated by the long-term findings from ACOSOG Z1031 [9]. The study randomized 510 clinical stage 2-3 postmenopausal women with strongly ER positive breast cancer to one of three aromatase inhibitors - exemestane, letrozole, or anastrozole. Of the patients who were thought to require mastectomy at time of presentation, over $50 \%$ were able to undergo breast conservation therapy. Additionally, local or regional recurrence occurred at an estimated 5-year cumulative incidence of $1.6 \%$. In line with these findings, it will be interesting to observe whether NET during the COVID-19 pandemic leads to an increased rate of breast conservation therapy.

This approach has been endorsed not only by the U.S. COVID 19 pandemic consortium [2] but internationally as well, particularly in those countries hardest hit early on by the pandemic. Buonomo et al described the impact of COVID 19 on Italy, with limitations on both breast cancer screening and surgery [10]. Breast cancer is the most common cancer in Italy, with over 50,000 cases diagnosed annually, and NET was recommended as a bridge until resources were again available for routine cancer care.

In April 2020, Marti et al in Madrid, Spain published similar recommendations for luminal type (hormone positive, HER2 negative) breast cancer during the height of the COVID 19 pandemic [11]. They provided a simple protocol that could be applied to most cases of hormone positive/HER2 negative breast cancers, noting that these cases could be handled by primary care physicians, thus minimizing specialty breast clinic visits and possible COVID 19 exposure for the patients, and allowing surgery to be safely postponed.

These changes in clinical management were quickly adopted, as outlined by a survey of the members of the European Breast Cancer Research Organization of Surgical Trialists (EUBREAST) in April 2020 [12]. In 10 days, 377 breast centers in 41 countries completed the questionnaire. There were significant modifications in management, including increased utilization of gene expression profiles to evaluate need for adjuvant chemotherapy, and the majority recommended NET for luminal-A cancers to allow postponing of surgery. The authors noted that an unexpected positive effect of the pandemic was to stimulate uptake of evidence-based improvements in care, which were being used previously in only a minority of centers, including genomic profiling and hypofractionated radiation therapy.

One of the deterrents to endocrine therapy in both the neoadjuvant and adjuvant setting has been the low adherence to medication regimens by patients. The reported completion rates for adjuvant hormonal therapy regimens are approximately $50 \%$ and have not been improved by educational or technology-based interventions [13]. Improving compliance with long-term adjuvant endocrine therapy is critical as patient acceptance is low. As demonstrated by our analysis of our institutional database, the unique situation posed by the current COVID-19 pandemic has resulted in a high initial acceptance of endocrine therapy $(80 \%)$.

Little information is available on premenopausal women, who make up $25 \%$ of this study group, and NET is not commonly recommended for this population. The available data suggest that NET treatment is effective in well-selected pre-menopausal women. Barbie et al reported on their single institution experience at the Washington University of St. Louis Alvin J. Siteman Cancer Center [14]. They identified 162 patients with stage I-III estrogen receptor-positive breast cancer treated with neoadjuvant ET between 2003 and 2012. Of this group, 17 patients were premenopausal, and 11 (65\%) had a response with a decrease in tumor size of at least $30 \%$. The STAGE Study results, released in 2012, reported that $70 \%$ of premenopausal women treated with anastrazole and goserelin had a partial to complete response during 24 weeks of therapy with low toxicity [15].

We are currently investigating this patient population 
further - evaluating length of use, as well as the psychosocial and behavioral factors that influence willingness to take endocrine therapy - and to apply these lessons to management of early-stage ER positive breast cancer. Hopefully this experience will influence practice and expand the role of NET and uptake of hormonal therapy once the pandemic resolves, rather than simply being a crisis-induced stop-gap measure.

\section{Conclusion}

The COVID-19 pandemic has presented an opportunity to apply the underutilized, yet effective, treatment strategy of NET in patients with ER positive DCIS and invasive breast cancer. Only time will tell if this experience will change our long-held approaches to early stage breast cancer [16]. Further exploration of our institution's database will allow us to track patients prospectively who have received NET, particularly with regard to their oncologic outcomes, and to identify factors which can lead to increased medication compliance.

\section{References}

[1] Liang W, Guan W, Chen R, Wang W, Li J, Xu K, Li C, Ai Q, Lu $\mathrm{W}$, Liang $\mathrm{H}, \mathrm{Li} \mathrm{S}, \mathrm{He}$ J. Cancer patients in SARS-CoV-2 infection: a nationwide analysis in China. Lancet Oncol. 2020 Mar; 21 (3): 335-337. doi: 10.1016/S1470-2045(20)30096-6. Epub 2020 Feb 14. PMID: 32066541.

[2] Jill R. Dietz; - Meena S. Moran; Steven J. Isakoff. Recommendations for prioritization, treatment, and triage of breast cancer patients during the COVID-19 pandemic. the COVID-19 pandemic breast cancer consortium. Breast Cancer Research and Treatment (2020) 181: 487-497.

[3] Cortazar P, Zhang L, Untch M, et al. Pathological complete response and long-term clinical benefit in breast cancer: the CTNeoBC pooled analysis. Lancet 2014; 384: 164-172.

[4] Morgan J, Wyld L, Collins KA, Reed MW. Cochrane Database of Systematic Reviews: Surgery versus primary endocrine therapy for operable primary breast cancer in elderly women (70 years plus) (Review). Cochrane Database of Systematic Reviews 2014, Issue 5. Art. No.: CD004272.

[5] Semiglazov V. F., Semiglazov V. V, Dashyan G. A, et al. Phase 2 Randomized Trial of Primary Endocrine Therapy Versus Chemotherapy in Postmenopausal Patients With Estrogen Receptor-Positive Breast Cancer. Cancer 2007; 110, 2: 244-255.
[6] Spring, L. M., Gupta, A., Reynolds, K., et al. Neoadjuvant Endocrine Therapy for Estrogen Receptor-Positive Breast Cancer: A Systematic Review and Meta-analysis. JAMA Oncol. 2016 November 01; 2 (11): 1477-1486.

[7] Chiba A., Hoskin T. L., Heins C. L., et al. Trends in Neoadjuvant Endocrine Therapy Use and Impact on Rates of Breast Conservation in Hormone Receptor Positive Breast Cancer: A National Cancer Data Base Study. Ann Surg Oncol. 2017 February; 24 (2): 418-424.

[8] Minami C. A., Kantor O., Weiss A., et al. Association Between Time to Operation and Pathologic Stage in Ductal Carcinoma inSitu and Early-Stage Hormone Receptor-Positive Breast Cancer. JACS. Vol. 231, No. 4, October 2020 pages 343-347.

[9] Hunt K, et al. Local-regional Recurrence (LRR) Following Neoadjuvant Endocrine Therapy (NET): Data from ACOSOG Z1031 (Alliance). SSO, Virtual Meeting August 17, 2020.

[10] Buonomo OC, Materazzo M, Pellicciaro M, Caspi J, Piccione E, Vanni G. Tor Vergata University-Hospital in the Beginning of COVID-19 Era: Experience and Recommendation for Breast Cancer Patients. In Vivo 2020; 34 (3 Suppl): 1661-1665.

[11] Marti C, Sanchez-Mendez JI. Neoadjuvant endocrine therapy for luminal breast cancer treatment: A first-choice alternative in times of crisis such as the COVID-19 pandemic. ecancer 2020; 14: 1027.

[12] Gasparri ML, Gentilini OD, Lueftner D, Kuehn T, Kaidar-Person O, Poortmans P. Changes in breast cancer management during the Corona Virus Diease 19 Pandemic: An international survey of the European Breast Cancer Research Association of Surgical Trialists (EUBREAST). The Breast 2020; 52: 110-115.

[13] Bickell NA, Wang JJ, Oluwole S, Schrag D, Godfrey H, Hiotis K, Mendez J, Guth AA. Missed opportunities: racial disparities in adjuvant breast cancer treatment. J Clin Oncol. 2006 Mar 20; 24 (9): 1357-62. doi: 10.1200/JCO.2005.04.5799. PMID: 16549830.

[14] Barbie T. U., Ma C., Margenthaler, JA. Management of Premenopausal Women with Neoadjuvant Endocrine Therapy: A Single-Institution Experience. Ann Surg Oncol. 2015 Nov; 22 (12): 3861-5.

[15] Masuda N, Sagara Y, Kinoshita T, et al. Neoadjuvant anastrazole versus tamoxifen in patients receiving goserelin for premenopausal breast cancer (STAGE): a double-blinded, randomized phase 3 trial. Lancet Oncol. 2012; 13: 345-352.

[16] Thompson CK, Lee MK, Baker JL, Attai DJ, DiNome ML. Taking a second look at neoadjuvant endocrine therapy for the treatment of early stage estrogen receptor positive breast cancer during the COVID-19 outbreak. Ann Surg. 2020; 272: e96-e97. 